Interventions (or Assessment of Risk Factors) Rider injury during EWS Enduro mountain biking.

Main Outcome Measurements Injury prevalence.

Results Overall $8.9 \%$ of riders were injured during the two EWS seasons (9.35 injuries per 100 riders) with on average 12.3 days time-loss per injury. Female rider injury prevalence was higher, but severity lower compared with male riders (11.3\%, 6.4 days; 8.7\%, 13.2 days, respectively). Two-thirds (64\%) of injuries occurred during racing, with $31.4 \%$ of injuries reported in inexperienced (1-race) riders. The shoulder/ clavicle $(13.3 \%$ of all injuries) followed by the hand $(9.0 \%)$ and head $(9.0 \%)$ were the most common affected anatomical locations, with concussion injury most frequent $(7.2 \%$ of all injuries) and shoulder/clavicle fracture causing the greatest burden (442 total days). Of those displaying concussion symptoms 29\% continued racing, of those diagnosed with concussion $43 \%$ reported no time-loss (i.e. time off) post-race. Conclusions The overall rate and severity of rider injury during EWS race events was low, but targeted injury prevention strategies, around shoulder injury pre- and rehabilitation, concussion education, and new/inexperienced rider qualification criteria may help to reduce the rate, severity and overall burden of some injuries.

\section{USING A MOTIFS INTERVENTION TO INFLUENCE PATIENT-REPORTED OUTCOMES: A RANDOMIZED CROSS-OVER PLAUSIBILITY STUDY}

${ }^{1}$ Niklas Cederström, ${ }^{2}$ Simon Granér, ${ }^{3}$ Gustav Nilsson, ${ }^{1}$ Eva Ageberg. 'Department of Health Sciences, Lund University, Lund, Sweden; 'Department of Psychology, Lund University, Lund, Sweden; ${ }^{3}$ Malmö Idrottsklinik, Malmö, Sweden

\subsection{6/bjsports-2021-IOC.341}

Background Injury prevention training focuses on physical execution of movement, but does not typically address mental aspects important for sport. A novel training model with integrated mental training, MOTor Imagery to Facilitate Sensorimotor Re-Learning (MOTIFS), is a potential method of addressing important mental and physical training aspects.

Objective To evaluate enjoyment of MOTIFS training. The primary hypothesis was that participants would report greater enjoyment following MOTIFS training than injury prevention (IP) training exercises.

Design Block-randomized 2x2 cross-over trial.

Setting Recreational Swedish athletes tested at a university lab. Patients (or Participants) Thirty athletes (18-31 years, 50\% women) currently or previously active in team ball sports were included. Participants with pain or injury preventing jump and/or directional changes were excluded.

Interventions (or Assessment of Risk Factors) MOTIFS training integrated equipment and sport-specific experiences into physical exercises to increase individualized realism and meaning. The IP training exercise condition included solely physical exercise.

Main Outcome Measurements The main outcome was the Physical Activity Enjoyment Scale (PACES). Secondary outcomes included Self-Assessment Manikin (SAM; subscales Valence, Arousal, Dominance), Borg's scale of perceived exertion (RPE), training duration, and maximum pulse.

Results PACES scores were better following MOTIFS training compared with IPT (mean diff 24.67; 95\% CI 30.34;-19.00). SAM Valence (median 2, quartiles 1-3), Arousal (median 1; quartiles 0-2.25), and Dominance (median 0.5; quartiles 0-2), as well as RPE (med 1; quartiles -0.25-2), and maximum (median 7.50; quartiles 0.25-16.75) pulse were higher following MOTIFS training. MOTIFS training took 5.34 minutes longer (95\% CI $-0.17 ;-0.73)$.

Conclusions Athletes perceived MOTIFS training as more enjoyable and reported more positive psychological responses than IP training exercises. Results indicate MOTIFS training is more fun and increases physical exertion, potentially improving IP training exercise quality and adherence by increasing athlete involvement and sport relevance. MOTIFS training took more time, so this should be taken into account during planning of exercises.

\section{THE TEST-RETEST RELIABILITY OF BILATERAL AND UNILATERAL FORCE PLATE DERIVED PARAMETERS OF THE COUNTERMOVEMENT PUSH UP (CMPU) IN ELITE GB BOXERS}

${ }^{1,2}$ Gemma Parry. ${ }^{1}$ English Institute of Sport, Sheffield, UK; ${ }^{2}$ The University of Salford, Manchester, UK

\subsection{6/bjsports-2021-IOC.342}

Background Upper limb muscular power output is a fundamental aspect across a number of explosive short duration sports such as boxing. Regular consistent production of high muscular power is a highly desirable characteristic and essential pre-requisite. Despite this importance, there is at present no gold standard test for upper limb force development performance, knowledge of normative performances is important for proper evaluation of risk factors for injury, return to play and programming.

Objective The aim of this study was to investigate the testretest reliability of force plate derived measures of countermovement push-up (CMPU) in Elite Boxers.

Design Test-Retest reliability approach.

Setting World Class Programme and Podium Potential Athletes within training environment.

Patients (or Participants) 18 Elite Great British (GB) Boxers participated within this study.

Main Outcome Measurements Kinetic data collected included peak force $(\mathrm{PF})$, mean force $(\mathrm{MF})$, flight time $(\mathrm{FT})$, rate of force development (RFD), Impulse and Vertical Stiffness.

Results No significant differences between the two trial occasions for any of the derived performance measures. Intraclass Correlation Coefficients (ICCs) indicated moderate to high reliability (ICC $=0.68-0.98$ ) and low co-efficient of variation $(\mathrm{CV}=3-10 \%)$, mean force demonstrated the greatest reliability $(\mathrm{CV}=3 \%)$.

Conclusions Reliable and purposeful methods of assessment are integral to understanding individual's performance potential but to also monitor training programme affects, long-term development and talent identification. Results highlight upper limb power output in elite level GB boxers can be reliably assessed by practitioners using force-plate and force-time derived parameters, and that unilateral data can be reliably extrapolated from the bilateral condition. When using CMPU to monitor training programme affects, no difference between limbs should be noted. This will be useful if completed prior to any injury, as if any deficits or subsequent injury occurs, CMPU can be used to better appraise and guide injury rehabilitation until the athlete returns to improved performance levels. 